\title{
Crack Characteristics and Their Importance to NDE
}

\author{
Mika Kemppainen • Iikka Virkkunen
}

Received: 23 September 2010 / Accepted: 13 May 2011 / Published online: 2 June 2011

(C) The Author(s) 2011. This article is published with open access at Springerlink.com

\begin{abstract}
The reliability of non-destructive evaluation depends on multitude of different factors. Consequently, it is difficult to assess the performance of the system. The only practical way to overcome this complexity and asses inspection reliability is using practical trials. In practical trials the inspection is performed on a known, flawed sample and reliability is judged by comparing the acquired inspection result with known state of the sample. However, confirming (and demonstrating), that the practical trials and, in particular, the used flaws are representative to postulated inspection case is, at present, challenging.

In this paper, the open literature is reviewed and compiled to the extent necessary for providing a starting point for confirming and demonstrating representativeness of flaws used in the practical trials. The available information on essential flaw parameters for various NDE techniques is reviewed. The used measurement methods for each parameter is discussed and the available in-service data summarized. Finally, a simple procedure is proposed for confirming and demonstrating the representativeness of used flaws.
\end{abstract}

Keywords Nondestructive evaluation - NDE . Qualification · NDE reliability · Nondestructive testing · NDT · Crack characterization · Crack characteristics

\section{Introduction}

The reliability of non-destructive evaluation depends on multitude of different factors. These range from physical aspects of the used technology (e.g, wavelength of ultrasound)

M. Kemppainen · I. Virkkunen $(\bowtie)$

Trueflaw Ltd., Tillinmäentie 3, tila A113, 02330 Espoo, Finland

e-mail: iikka.virkkunen@trueflaw.com to application issues (e.g. probe coupling or scanning coverage) and human factors (e.g. inspector training and stress or time pressure during inspection). Due to this complexity, the only practical way to asses inspection reliability and to confirm that the inspection procedure functions as intended is by using practical trials. In practical trials the inspection is performed on a known, flawed sample and reliability is judged by comparing the acquired inspection result with known state of the sample. In order to get reliable performance data from practical trials, it is paramount, that the inspection arrangement and flawed sample used are representative to real inspection situation.

However, confirming (and demonstrating), that the used artificial flaws are representative to postulated inspection case is, at present, challenging. The methods used to define and measure various crack characteristics vary. Thus, compiling cohesive justification for used flaws is laborious and difficult. Furthermore, there is no standard or best practice to follow on how to compile and present such information.

In this paper, the open literature is reviewed and compiled to the extent necessary for providing a starting point for confirming and demonstrating representativeness of used artificial flaws. The available information on essential parameters for various NDE techniques is reviewed. The used measurement methods for each parameter is discussed and compared and the available in-service data summarized. Finally, a simple procedure is proposed for confirming and demonstrating the representativeness of used flaws.

\section{Representativeness of Flaws}

Representativeness, in this context, means, that the used artificial cracks give similar response in the used NDE than actual, service-induced cracks would. This, of course, is not trivial to confirm or to demonstrate. 
One approach, that has been used, is to directly compare NDE response acquired from artificial flaws and serviceinduced cracks. This approach, however, has several significant drawbacks and thus cannot be recommended. Firstly, the result becomes overly linked to the used NDE method. If the method is developed or changed even slightly, the response may change (this is generally the aim and reason for the development) and thus the comparison must be re-done every time. Secondly, representative service-induced cracks are not generally available; this is the motivation for the use of artificial flaws in the first place. If representative serviceinduced cracks of known size were available, artificial flaws would not be needed as these service-induced cracks could be used in stead. Thirdly, service-induced cracks, as any natural phenomena, exhibit wide range of variance in their characteristics. Thus, confirming similar signal-response to limited set of service-induced cracks (when available) does not confirm expected behavior if the actual service-induced cracks differ from the used sample due to natural variance.

The second approach that can be used, is to show representativeness implicitly by used production method. That is, the artificial flaw is produced by the same damage mechanism that is postulated to be active in service and thus, it is argued, the response must be similar. This is far better than comparing signal response since it does not require availability of representative service-induced cracks (only the postulated cracking mechanism must be known). However, it is not always possible to produce artificial cracks to relevant components with the postulated damage mechanism. Furthermore, it is a bit unclear how broadly "damage mechanism" may be defined while still retaining sufficient representativeness. Still, where available, this approach provides a simple and solid justification for representativeness of used cracks.

The third and the recommended approach is to use metallographic crack characteristics as the basis for representativeness. The wide variety of natural cracks is broken down to determine discrete set of crack characteristics. The magnitudes of such essential characteristics are measured from service-induced cracks and from artificial cracks. Then, the used NDE methods are studied to define the list of characteristics that are essential for this particular method. Finally, the values of essential crack characteristics (for particular NDEmethod) are compared between literature data from service induced cracks (with postulated defect mechanism(s)) and artificial cracks. Representativeness is confirmed by demonstrating that the used artificial cracks are similar to postulated service-induced cracks in terms of the defined essential crack characteristics. This approach allows solid justification for representativeness of used cracks while retaining as much flexibility in flaw production as possible. By narrowing or widening the selection of the essential parameters, used cracks can be chosen to serve wider range of NDEmethods and/or crack types. Also, if certain characteristic can't be reproduced by the used flaws, this approach brings this into light so that it can be addressed by, e.g., technical justification or additional set of test samples.

\section{Essential Characteristics for NDE}

Wide variety of different NDE methods is available and new ones are constantly developed. Thus a comprehensive list of essential crack characteristics for every method cannot be compiled by one party but the final definition of essential characteristics to be considered must be done in collaboration with the inspection vendor when all the details of the used inspection technique are know. However, to provide general background work for such analysis, information available on the open literature is summarized below. Also, if there is uncertainty about the significance of certain crack characteristic, it may always be added to the list of characteristics to be reproduced by the used artificial flaws. Thus, representativeness of used cracks can always be shown, even in the absence of detailed information from used NDE-method (with the possible risk of using "overly representative" cracks).

In the current paper, the focus is on ultrasonic techniques. The reason is that of all the critical inspection techniques used to detect and size real cracks, ultrasonic inspections are most widely used. Furthermore, sensitivity of the ultrasonic techniques to different crack characteristics is known and recognized by the international NDE community.

\subsection{Flaw Properties Affecting Ultrasonic Detection and Sizing}

There is a lot of experience confirming the difficulty to reliably detect and size service-induced thermal fatigue cracks as reported, e.g., by Edwards et al. [6, 7], Pirson et al. [15], and Gauthier [8]. The difficulty of the inspection is caused by typical characteristics of cracks, which affect, e.g., propagation, reflection, diffraction, transmission, attenuation and diffusion of ultrasonic energy $[2,10]$. Such flaw characteristics have been stated to be, amongst others, location, orientation and size of a crack (e.g., [19]), the opening of a crack and crack tip (e.g., [1, 20, 24]), the remaining residual stresses in the material (e.g., $[8,11])$, fracture surface roughness (e.g., [13, 20]), plastic zone (e.g., [16]), and filling of the crack with some substance (e.g., [2]).

\subsubsection{Effect of Fracture Surface Roughness on Detection and Sizing}

The fracture surface of a realistic flaw is not ideally planar, but it has natural irregularities. The general effects of the fracture surface roughness of a reflector on the spatial distribution of scattered waves are well known and have been 

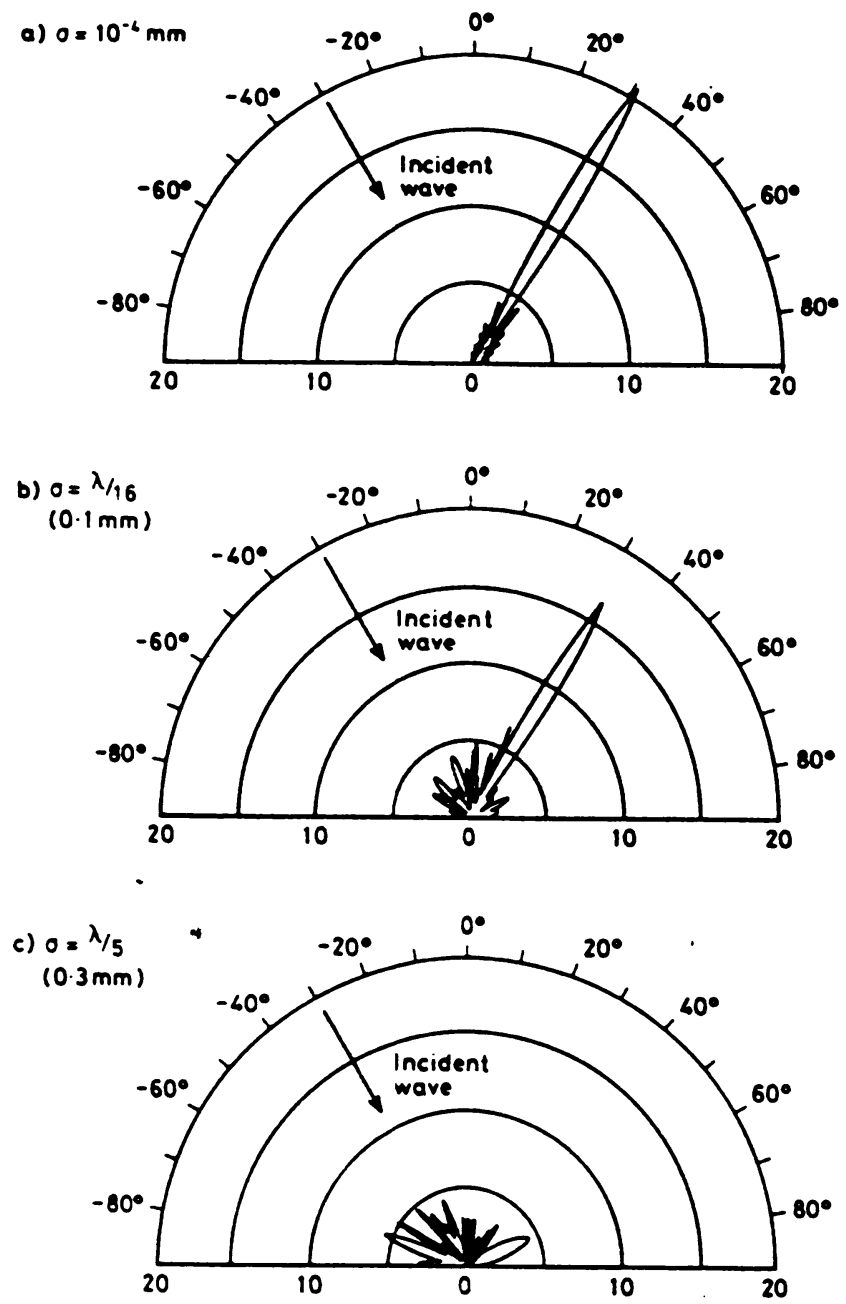

Fig. 1 Polar plots of scattered amplitude distributions from surfaces of different roughness values $(\sigma)$, when a $2 \mathrm{MHz}$ monochromatic wave is incident at $30^{\circ}[13]$

widely studied both practically and theoretically. The principle is that by increasing the reflection surface roughness, the forward scattered high amplitude is decreased and energy is redistributed into a more widely spread diffuse field $[13,20]$. Figure 1 shows an example of the effect of surface roughness (from smooth to very rough) to the distribution of the scattered field. From a smooth surface a strong coherent field will arise because of the interference between all the scattered wavelets from all parts of the surface. Enhanced surface roughness destroys the summation as the phase of the wave varies with position along the flaw surface. Thus, the strength of the coherent field is reduced and a diffuse, widely scattered field of varying phase will be generated.

Increased surface roughness reduces the detection sensitivity of the methods relying on specular signal. Off-specular signals may be increased because of the diffuse field. This effect was seen for certain inspection geometries in the PISC-II exercise, where small rough flaws were found to have higher detectability than the smooth flaws of the same size. Increased frequency of the used probe increases the scattering effect due to fracture surface roughness [2]. Furthermore, if the surface has a regularly shaped fracture surface roughness profile, reflection of the incident sound wave may favor certain directions thereby decreasing the testing repeatability and reliability [9]. Effect of surface roughness on the scattered energy can be used qualitatively in flaw characterization to separate smooth planar flaws, rough planar flaws and volumetric flaws [3, 13].

Theoretical models and experimental work on the effect of surface roughness have shown quite good agreement with smaller surface roughness, but with increased values this is not the situation. For example, Ogilvy [13] found an agreement between model predictions and experimental results to be typically within $3 \mathrm{~dB}$, except for very rough surface where the theoretical prediction results did not agree with the experimental results.

The effect of fracture surface roughness on the detectability becomes advantageous, when the flaw is tilted. When the incident wave is normal to the reflection surface, the specular signal is detected and increased surface roughness decreases the amplitude hence decreasing the detectability. When the flaw is tilted, the increased surface roughness allows reflection of ultrasonic energy in different directions, as theoretically modeled by Ogilvy [13]. This means that by increased tilt the detection changes from specular to offspecular field. For example, Toft [18] showed experimentally that increase of the fracture surface roughness of the flaw decreases the signal amplitude of well-oriented flaws (Fig. 2). Furthermore, increase of the tilting angle decreases the signal amplitude, but with increasing surface roughness the rate of amplitude decrease is diminished. Through tilting the flaw, the signal amplitudes from rough flaws exceed those from smooth flaws as detection moves out from the main lobe of specular energy to the diffuse field.

The level of misorientation, after which the detection is enhanced, depends on the flaw size and shape. The larger is the flaw, the smaller is the degree of mis-orientation beyond which the surface roughness will enhance detection. It must be noted that, since the diffuse field amplitude will never exceed the amplitude of the coherent field, the detectability can be enhanced only with sufficiently large misorientation. The model predictions showed this to be more than $20^{\circ}$ from normal angle $\left(0^{\circ}\right)$ [13].

However, there are studies that do not clearly indicate the effect. For example, Yoneyama et al. [24] studied the effect with conventional $45^{\circ}$ and focusing $45^{\circ}$ and conventional $60^{\circ}$ and $70^{\circ}$ probes. In the experimental work they used mechanical fatigue cracks with maximum surface roughness values $\left(R_{y}\right)$ varying from $40 \mu \mathrm{m}$ to $70 \mu \mathrm{m}$ and average values $\left(R_{z}\right)$ from $34.6 \mu \mathrm{m}$ to $55.9 \mu \mathrm{m}$ (larger with deeper cracks). The difference in crack corner echo height was compared to the echo from a smooth corner. Authors 


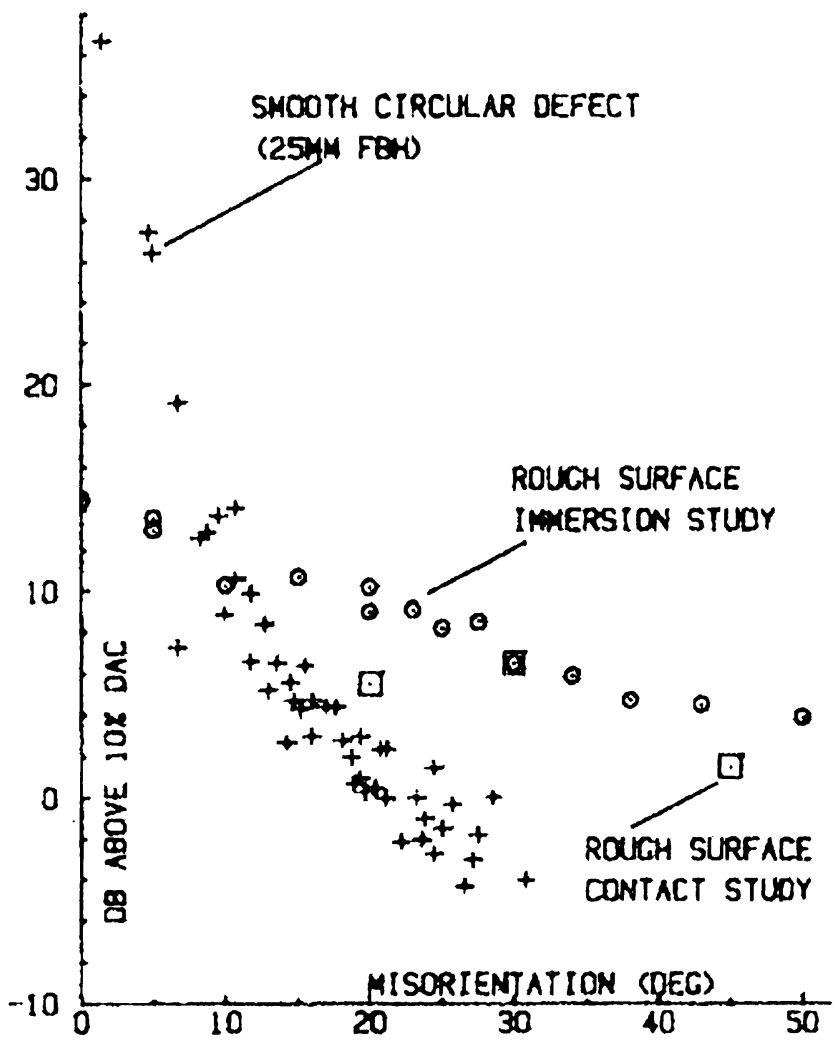

Fig. 2 Signal levels of rough and smooth flaws as a function of flaw misorientation [18]

found some differences between the echo heights from the smooth corner and corners of different fatigue cracks, but they considered them negligible and drew a conclusion that the difference of surface roughness between an EDM notch and a mechanical fatigue crack may be neglected when conducting flaw detection.

Amplitude differences at different locations of flaws are used to size flaws with techniques relying on amplitude changes at flaw extremities [13]. With smooth flaws, the signal amplitude drops clearly near the edges of the flaw. However, for rougher flaws there is not a clear plateau region from which the decibel drop could be measured, but the signal amplitude fluctuates across the flaw. The principle and difference between smooth and rough flaws is shown in Fig. 3.

Surface roughness of the flaw affects also sizing techniques relying on the time-differences from flaw extremities [13]. Two clearly separate pulses are detected from a smooth flaw, from which the flaw size can be calculated. These distinct pulses arise from edge diffraction. From a rough flaw, a continuous pulse may be obtained which is a superposition of the edge-diffracted pulses and diffuse scattering from all parts of the surface. Roughness may cause a loss of distinct diffracted pulses and hinder the timing measurements. The principle of the effect is shown in Fig. 3.
Smooth Flaw

Rough Flaw

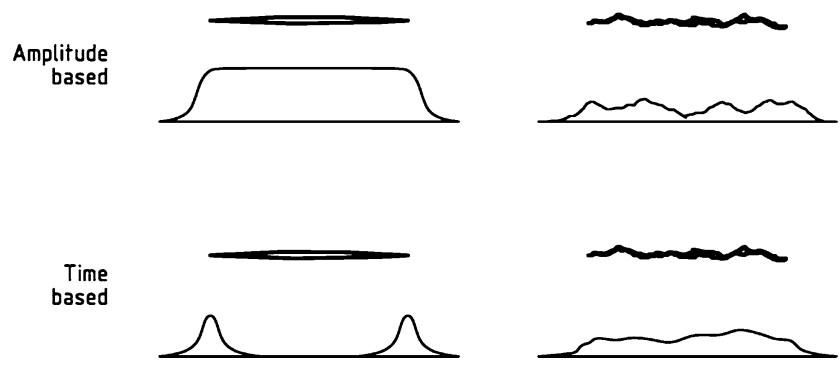

Fig. 3 Principle of the effect of flaw surface roughness on the amplitude- and time-based sizing techniques

\subsubsection{Effect of Opening and Residual Stresses on Detection and Sizing}

Basically, with increasing crack opening the obtained ultrasonic echo amplitude increases and when the crack is closed, echo amplitude decreases. In the following the effect of flaw opening to the obtained ultrasonic response is introduced. Furthermore, the condition of residual stress is treated separately as an individual factor affecting the flaw opening and obtained ultrasonic response.

The recorded amplitude changes are related to the reflection surface movements. If the crack is open through its full length, the whole surface from the crack opening corner to the crack tip can vibrate freely as excited by the incident ultrasonic energy. If the crack is closed partly or through its whole length, the fracture surfaces pressed together hinder the free oscillation of the reflection surface. In this case, fracture surface heights touching each other allow transmission of the ultrasonic energy. Hence, the ultrasonic wave does not meet any boundary of different acoustic impedances. Vice versa, if the crack is open, there is a clear boundary of different acoustic impedances where reflection and scatter of the ultrasonic energy occur. By a continued increase of the flaw opening, a saturation level of the echo amplitude is reached where the reflection from the fracture surface is at maximum. For a mechanical fatigue crack, Iida et al. [11] reported a saturation level to be at $10 \mu \mathrm{m}$ width of the crack surface opening. When closing a crack, it is defined acoustically closed, when a large change in the ultrasonic signal is observed. However, according to Ibrahim et al. [10], the signal does not necessarily disappear completely.

The effect of flaw opening to the detection sensitivity of different mechanical fatigue cracks has been studied, e.g., by Yoneyama et al. [24]. Yoneyama et al. [24] loaded three different mechanical fatigue cracks by mechanical tensile and compressive loads to study the effect of crack opening on the obtained crack corner echo height. In their results, the echo height from crack opening significantly changed 


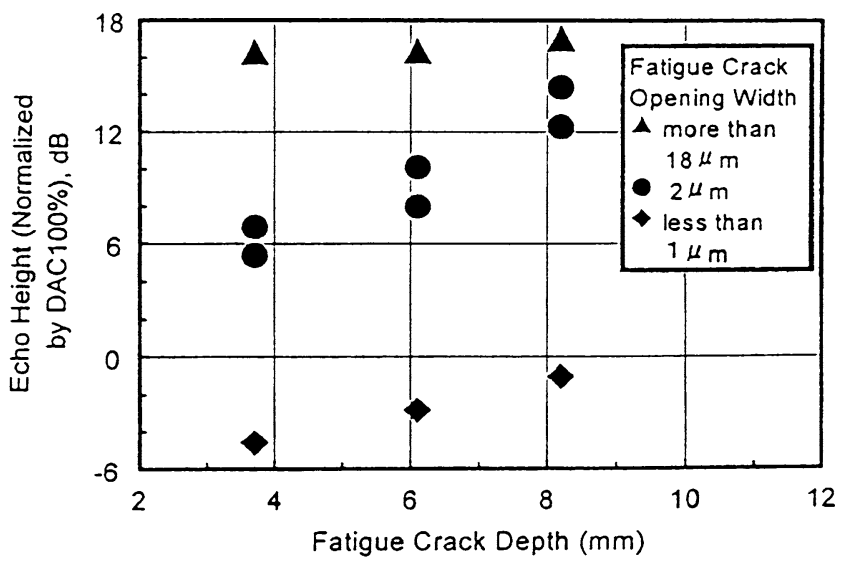

Fig. 4 Relation between fatigue crack surface opening width and echo height of three different sizes of cracks $(3.7 \mathrm{~mm}, 6.1 \mathrm{~mm}$ and $8.2 \mathrm{~mm})$ [24]

with the changed surface opening width. Furthermore, echo height with the same opening width increased with increasing crack depth (Fig. 4) finally being, with the largest opening widths $(>18 \mu \mathrm{m})$, approximately the same as what was obtained from an EDM-notch. The authors attributed their results to the effect of crack opening width, but they did not discuss the reasons for this. The results may only reveal the effect of different cross-sectional areas taking part to the reflection of the acoustic energy.

In the studies of detection sensitivity of mechanical fatigue cracks the effect of material condition to the interdependence of the opening of the crack and obtained ultrasonic echo amplitude height have been studied, e.g., by Becker et al. [2]. Ultrasonic amplitude height obtained from mechanically loaded fatigue cracks showed different behavior with cold-worked and annealed materials. In the coldworked condition, the amplitude height decreases gradually, when the crack is closed (compressive loading is increased). After annealing the sample, the change in amplitude height is much faster, i.e., the slope of amplitude change vs. applied load is steeper than that of the cold-worked sample. This was attributed to the material condition as in the coldworked material the crack tip closes first and after that gradually the rest of the crack, and the last part to close is the crack mouth. This is seen in the change of the ultrasonic amplitude first as a slow decrease, when the less reflective crack tip closes, and then as faster decrease when the rest, more reflective parts of the crack close. In the annealed material, the whole crack closes practically at the same time. This difference was attributed to the compressive residual stresses present near the crack tip of the cold-worked specimen causing immediate increase of the compressive stress from the beginning of the compressive loading. In the annealed material sample there is no stress near the crack tip as unloaded. Furthermore, the total signal drop was with the cold-worked material $32 \mathrm{~dB}$ and with the annealed material
$22 \mathrm{~dB}$, and both were reached at the yield stress of the material. Becker et al. [2] attribute the difference in the total amplitude drop and the magnitude of the drop to the force the fracture surfaces bear. In the annealed material much less force is available, because the yield strength of the annealed material is lower, being half of that of the cold-worked material.

As a separated factor of the crack opening, the present stress state affects the echo amplitudes obtained from the crack opening corner, fracture surface and crack tip. Hence, the results from the open literature indicated here do not only show the crack opening differences, but also the effect of the present stress state. The effect of the stress state is handled as the effect of residual stresses in the following. This is the case also during the in-service inspection, where the present stresses are not dynamic but static.

The possible present residual stresses affect the detectability of a flaw, as tensile residual stress opens and compressive residual stress closes the flaw. Studies on the effect of residual stresses have been performed experimentally with external mechanical loading, e.g., by Iida et al. [11], Yoneyama et al. [24], Becker et al. [2], Ibrahim et al. [10] and Denby et al. [5]. Theoretical studies on this topic have been published, e.g., by Temple (1985) and Wirdelius (1992). Denby et al. [5] mentioned that the reflection from fatigue flaws is most seriously affected by the compressive stresses, of all the flaws. Furthermore, flaw tips of the thermal fatigue flaws are considered to be the most challenging ones as the flaw tips are surrounded by a plastic zone already under compression.

The detectability is affected so, that the increasing tensile stresses (opening the crack) increase the echo amplitude and increasing compressive stresses (closing the crack) decrease the amplitude. With high enough loading, there will be a plateau in the amplitude value both in tension and compression. Similar phenomenon has been found for carbon steels [11] and austenitic stainless steels [2]. With high enough tensile loading the echo amplitude obtained from a mechanical fatigue crack may be almost identical to the one obtained from an EDM-notch [24].

The effect on the echo amplitude, and detection sensitivity, may be dependent on the direction of the load change, i.e., increasing or decreasing loading. As, in addition to plateau values obtained, a clear hysteresis may be recorded in the change of the ultrasonic amplitude during cyclic loading of a crack. This was shown by Becker et al. [2] with a mechanical fatigue crack in an austenitic stainless steel. During cyclic loading between tension and compression, the first loading to tension did not change the height of the signal amplitude from the unloaded condition. However, by the followed high enough compressive stress, the amplitude height dropped markedly, finally stabilizing at a lower plateau at loads well over the yield stress of the material. 
During the second load cycle (unloading-tension-unloadingcompression), while unloading from compression, the signal remained in the lower plateau, beginning to increase at a lower force than required to reach the plateau. In maximum tension, the same maximum value of the amplitude height was reached as in the first cycle. When the sample was loaded again in compression, lower force was needed to keep the lower plateau. Becker et al. [2] attributed the seen hysteresis in the change of the amplitude height to the plastic deformation taking place during the first compressive loading cycle.

The detection sensitivity is differently affected by loading with mechanical fatigue and thermal fatigue cracks. Even

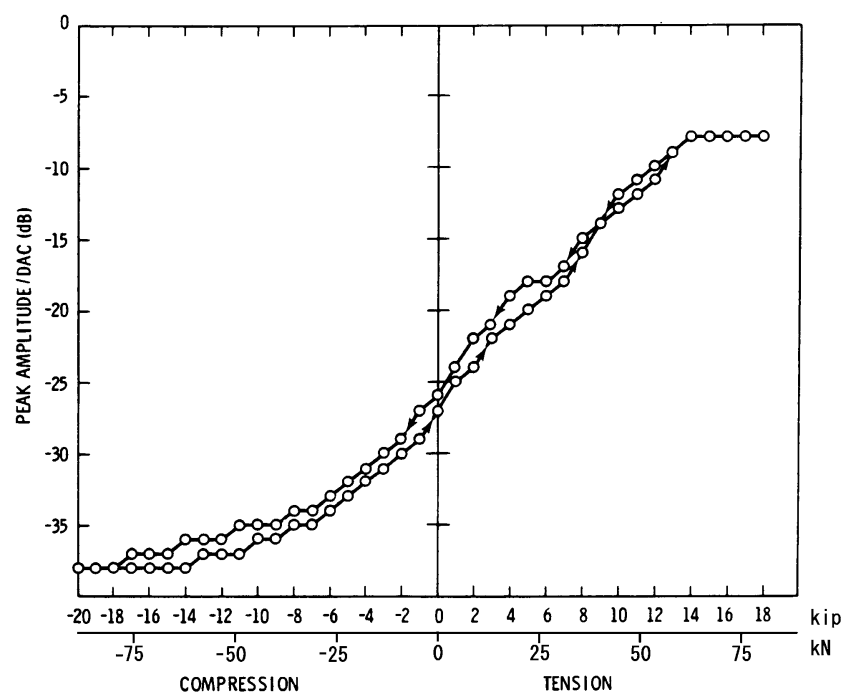

Fig. 5 Different stress conditions affecting the obtained ultrasonic echo height from a thermal fatigue crack in AISI 304 type austenitic stainless steel [2] though the basic phenomenon of ultrasonic echo amplitude change under cyclic loading is similar, there are some important differences. Typically, thermal fatigue cracks show low values of echo amplitude. Becker et al. [2] reported that thermal fatigue cracks showed low values of echo amplitude already when no external load was applied. As Fig. 5 shows, by application of an external load the thermal fatigue crack followed the theoretical behavior of reflection amplitude as a function of crack opening (the theoretical amplitude curve is shown in Fig. 6). That is, when the crack is under tension the plateau of high amplitude is obtained and under compression the low amplitude plateau of reflection is observed. When no external load is applied, the obtained echo heights lie in the lower part of the steep slope region between the plateaus. Consequently, unloaded thermal fatigue cracks are very sensitive to changes in the loading conditions. This is a result of the crack tightness and rough fracture surfaces allowing the surface heights to be, partly already as unloaded, in contact. Apart from this, mechanical fatigue cracks exhibit higher echo amplitudes as unloaded, hence being more detectable.

Although unloaded thermal fatigue cracks were undetectable (with 50\% DAC criterion), the application of the tensile stress equal to the yield strength made most of them detectable. Detection of cracks with such tightness in service conditions substantially depends on stress condition. If cracks are filled with water, according to Becker et al. [2], they can be undetectable under any realistic stress conditions as a result of the better acoustic energy transfer ability of water than air.

The obtained ultrasonic echo height, and detection sensitivity, under different stress conditions is also affected by the fracture surface roughness (i.e., planarity) of the flaw.
Fig. 6 Theoretical ultrasonic reflection coefficient as a function of distance between parallel stainless steel plates separated by air and water $(2.25 \mathrm{MHz}$, transverse wave at $\left.45^{\circ}\right)[2]$

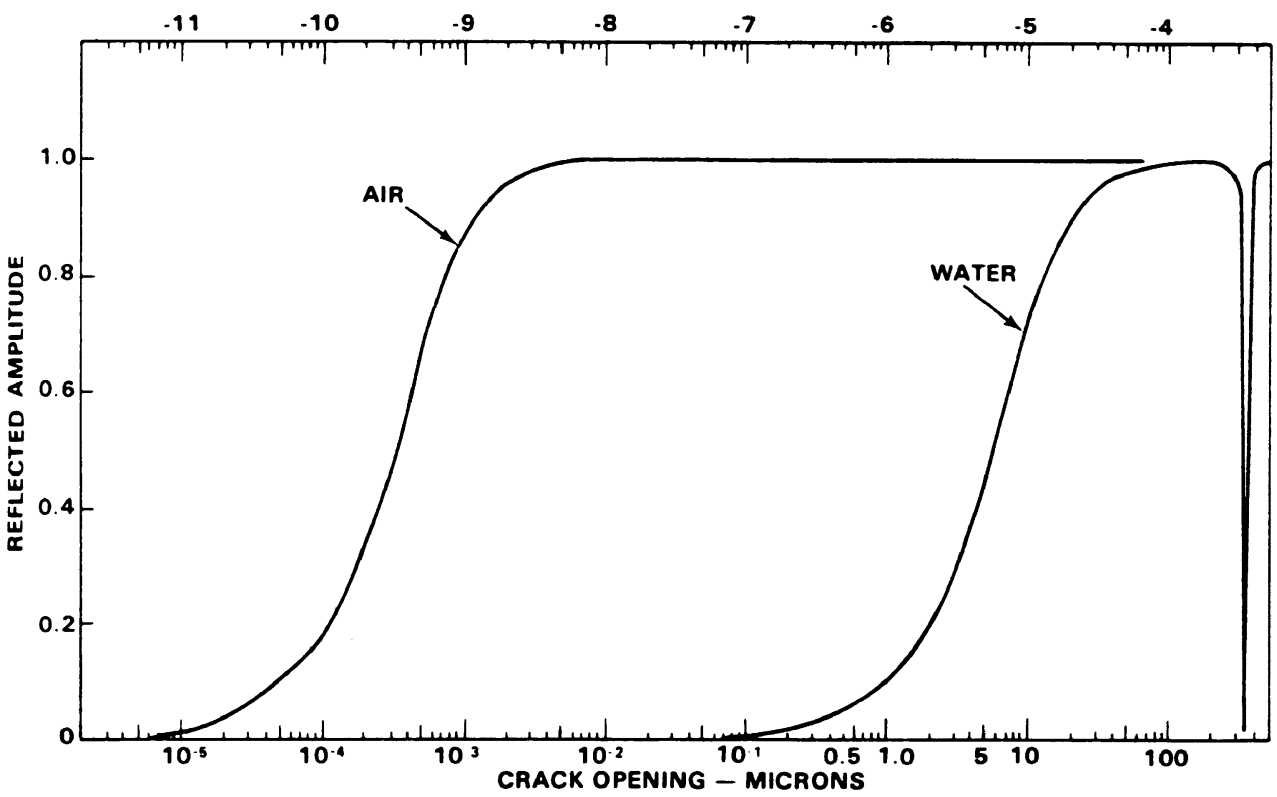


Fig. 7 Energy reflection coefficient of $10 \mathrm{MHz}$ longitudinal waves incident at (a) $20^{\circ}$ and (b) $30^{\circ}$ to the normal on the model crack under compressive stress [17]
Fig. 8 Energy reflection coefficient for longitudinal waves at normal incidence $\left(0^{\circ}\right)$ on rough cracks (three different rms values) under (a) $60 \mathrm{MPa}$ and (b) $160 \mathrm{MPa}$ compressive stress [17]
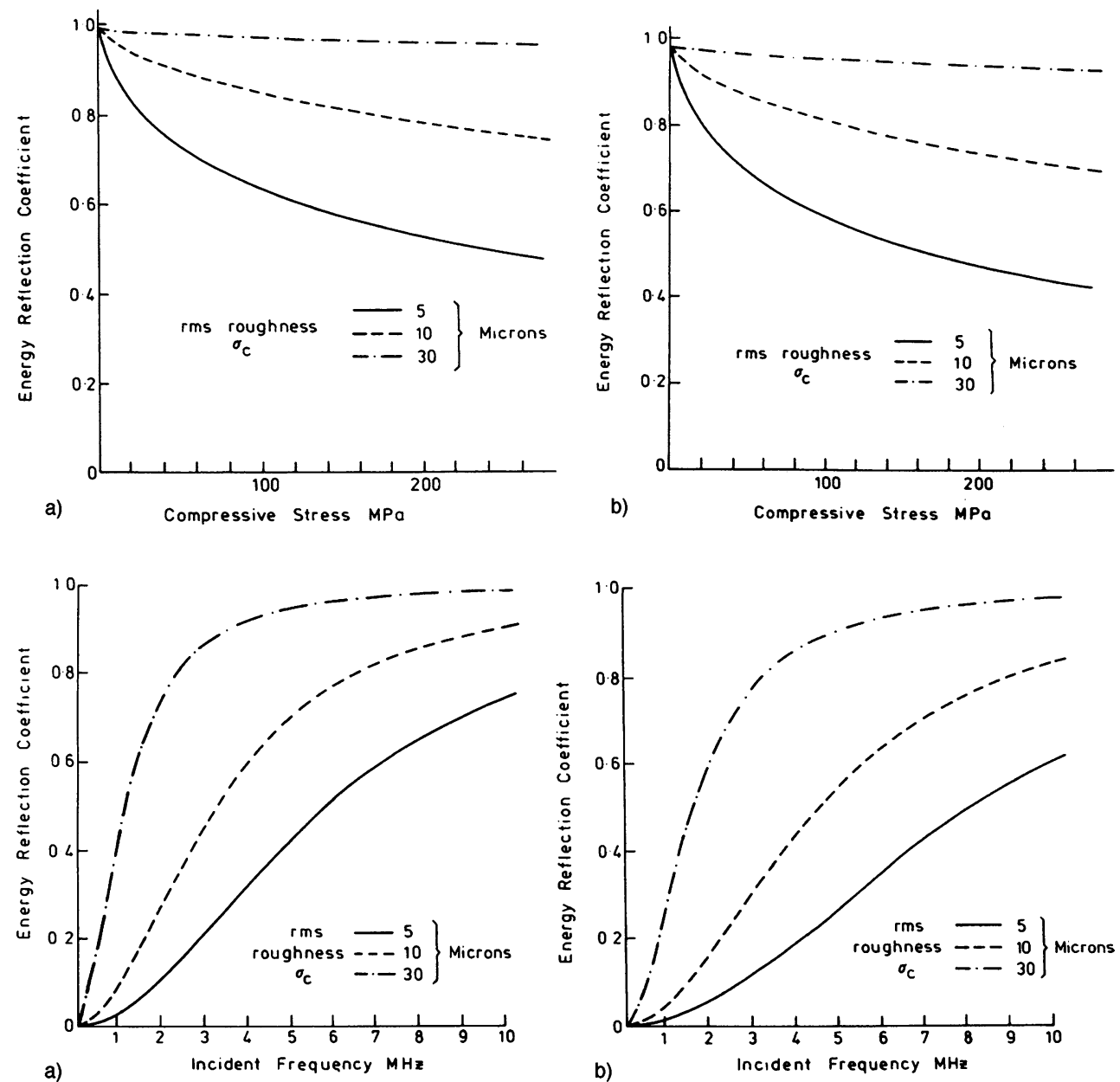

b)

ently the obtained echo amplitude, when the flaw tilt angle is varied. In the specular reflection (Fig. 9a), the signal level is decreased until the background pressure reaches $200 \mathrm{MPa}$. In normal incidence (Fig. 9b), the tip-diffracted signal is dropped from the signal level of an open flaw markedly already with $50 \mathrm{MPa}$ background pressure and increase to $200 \mathrm{MPa}$ decreases the amplitude only a bit more.

The effect of stress state and tilt angle to detection sensitivity was experimentally studied by Ibrahim et al. [10] resulting in differences in the echo heights of different incident beam angles under different compressive stresses. Ibrahim et al. [10] studied pulse-echo response from crack opening corners of three different mechanical fatigue cracks and, hence, their results are not directly comparable to the theoretical results given in Fig. 9. However, the tendency shown in Fig. 10, that the highest detectability is achieved with $45^{\circ}$ probe, was also shown by Wirdelius et al. [21]. The good results of $45^{\circ}$ probe were attributed to the favored orientation of large portion of small facets on the flaw fracture surface.

Sizing of the flaw, similarly than detection but probably with even stronger influence, is affected by the combined effect of the flaw opening width, residual stresses and material condition. However, there are no studies showing the elius [21] show that the background pressure affects differ- 
Fig. 9 Theoretical evaluation of pulse-echo signal responses for a penny-shaped flaw (depth $60 \mathrm{~mm}$, diameter $4 \mathrm{~mm}$ ) at different tilts and under different background pressures, with a zero degree, $3 \mathrm{MHz}$ longitudinal wave probe. Different line types indicate different stresses: $200 \mathrm{MPa}(---), 50 \mathrm{MPa}(-\cdot \cdot-)$ and unloaded open crack (-). Tilts were (a) $0^{\circ}$ (parallel to scanning surface), (b) $90^{\circ}$ (perpendicular to the scanning surface) and (c) $30^{\circ}$ [21]

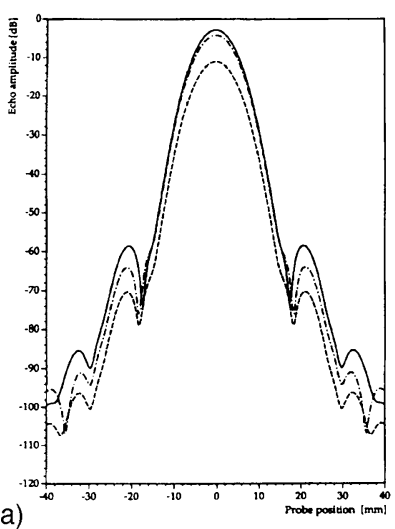

a)

b)
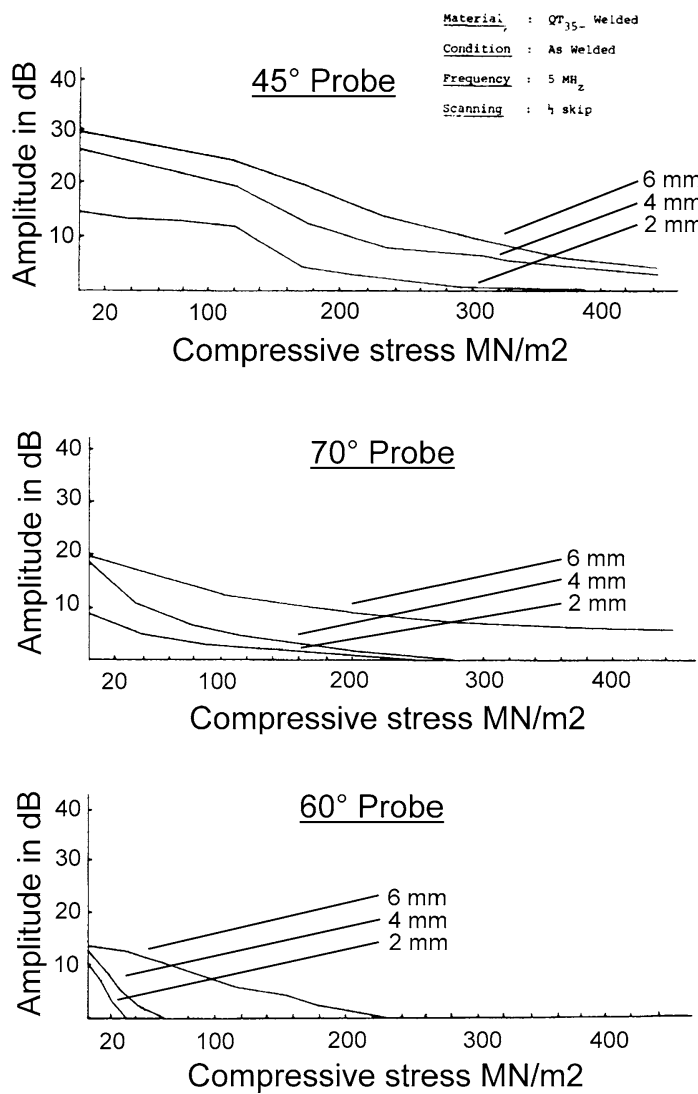

Fig. 10 Maximum signal responses from three different fatigue cracks as a function of compressive stress with three different $5 \mathrm{MHz}$ angled transverse wave probes [10]

effect of different flaw tip openings under different loading conditions, as was the case with detection sensitivity studies. Hence, all the sizing sensitivity studies are connected to different opening widths at the flaw mouth.

The effect of material condition to the obtained ultrasonic echo height from the flaw tip, i.e., sizing sensitivity, under different loading conditions has been studied, e.g., by Becker et al. [2]. The reported difference in the rate of change of the ultrasonic response from loaded crack tips of cold-worked and annealed materials was attributed to the
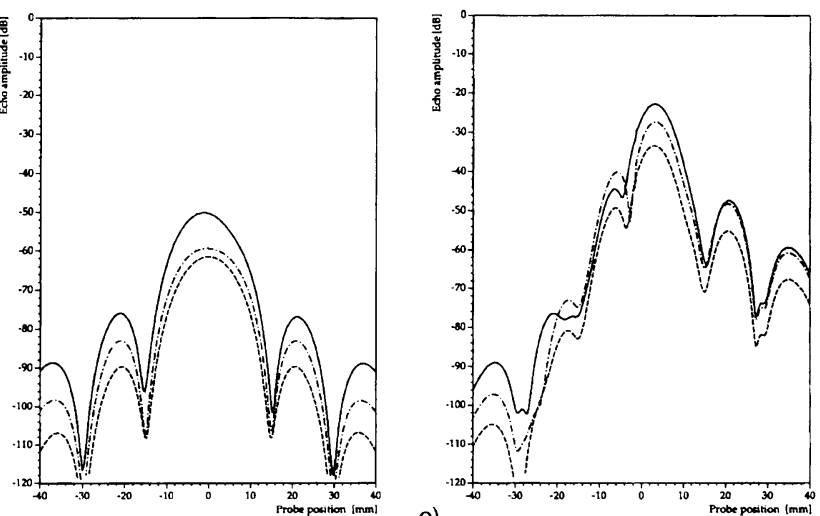

c)

different condition of residual stresses in the material. This was seen as slower change of obtained amplitude height with the cold-worked material and faster change with the annealed material. This difference was attributed to the first closing crack tip with the cold-worked material, while with the annealed material the faster amplitude drop indicates that the whole crack closes practically at the same time.

The growth of a fatigue crack always induces a plastic zone in the material around the crack tip, affecting the sizing sensitivity. With austenitic stainless steels the radius of the crack tip plastic zone can be from some hundred micrometres to some millimetres, depending on the loading used during the crack growth. The residual stresses inside the plastic zone are compressive, caused by the plastic tensile loads in front of the crack tip during crack growth. Such compressive stresses around the crack tip promote closure of the crack tip.

In addition to the plastic zone around the crack tip, a plastic wake forms at the fracture surfaces of the crack in any material during fatigue crack growth. By annealing the material, the plastic areas are stress relieved resulting in stress-free material. Consequently, the echo amplitude from the crack and crack tip will behave differently during loading. However, although there are differences between coldworked and annealed materials, the fatigue crack tips in both materials are very tight and sharp. They give, already as unloaded, a very weak ultrasonic response making the flaw sizing a difficult task. In case some loads are applied; the crack tip response is changed and if the loads are compressive they will result in marked difficulties in flaw sizing as the obtained amplitude is decreased.

The effect of residual stress on the flaw sizing sensitivity and accuracy has been studied, e.g., by Iida et al. [11] and Temple [17]. In these studies, test specimens containing different types of flaws were mechanically loaded. Studies were performed with austenitic and ferritic steels under different stress conditions both with metallic fracture surfaces and surfaces covered with oxide layers. Ultrasonic measurement methods used were, amongst others, based on crack tip reflection and diffraction. 


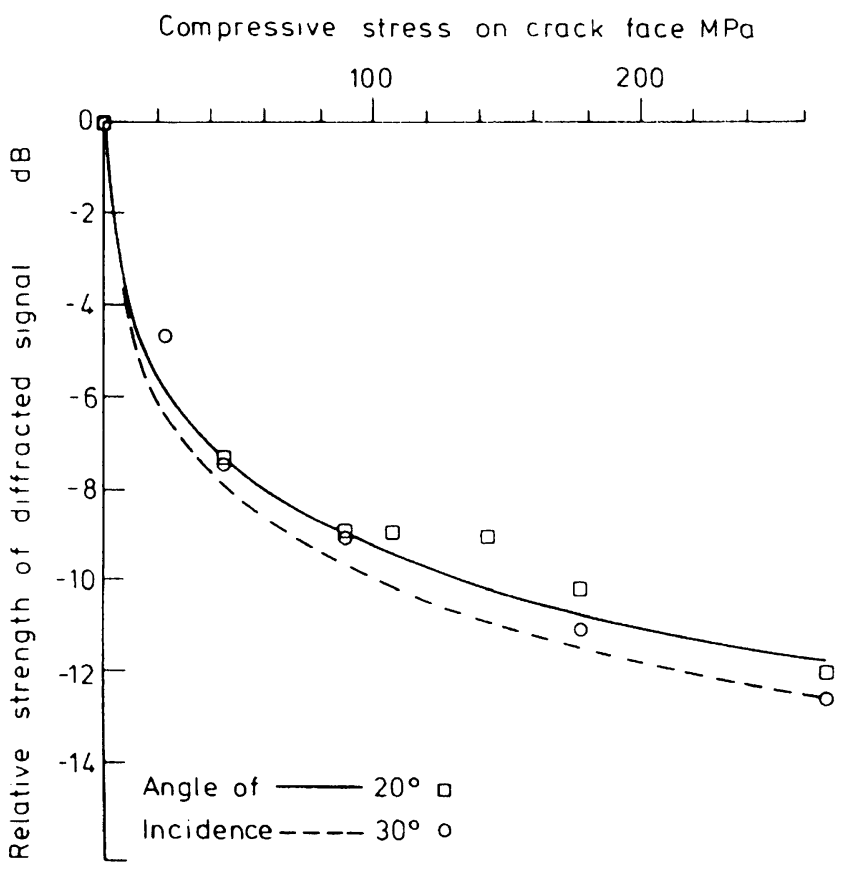

Fig. 11 Interaction between compressive stress and relative strength of crack tip diffracted signal [17]

The sizing sensitivity is reduced as the obtained amplitude height from the crack tip is decreased with increasing compressive stress. However, the lateral scanning graphs of flaw tip reflection show similar shape under different stress states. On the contrary, the flaw tip echo height is increased by increased tensile stress. According to Iida et al. [11] the sizing accuracy of DAC\% method showed more sensitivity to stress changes than the amplitude drop method. The flaw tip reflection is measurable for deeper flaws, but that may not be the case with shallow flaws. Iida et al. [11] mentioned the threshold depth to be $0.9 \mathrm{~mm}$; with the smaller flaws they could not measure the tip reflection echo even under tensile loading.

The sizing sensitivity is reduced by increased compressive stresses also with flaw tip diffraction based sizing techniques. With these methods the strength of the crack tip diffracted signal show clear correlation to applied compressive stress. Temple [17] has published results of correlation between theoretical calculations and experimental studies of stress vs. flaw tip diffraction (Fig. 11), showing reduction in the strength of the diffracted signal with increasing compressive stress. However, in the studies of Temple [17] the diffracted signal was not completely lost even with the maximum applied compressive stress. The maximum loss of signal strength was about $13 \mathrm{~dB}$ with maximum applied stress of $260 \mathrm{MPa}$.

More recently Packalén et al. [14] and Kemppainen et al. [12] studied the effect of crack opening on ultrasonic sizing on a set of artificial cracks that were carefully destructively analyzed after inspection. They confirmed that the magnitude of the crack opening affects the amplitude of the crack tip signal in that smaller opening gives smaller signal amplitude. This has direct consequence on sizing performance: with decreasing signal amplitude, the likelihood of misinterpretation in identifying of the crack tip signal increases. Similarly, with increasing noise amplitude (for flaws near weld), the likelihood of misinterpretation in identifying crack tip signal increases.

\subsubsection{Effect of Crack Orientation on Detection and Sizing}

Detection is changed from specular to off-specular field as the tilt of the flaw increases [13]. Especially with flaws having rough fracture surfaces, the effect of tilt angle on the detectability is pronounced.

When detecting flaws, the inspection performed in normal $\left(0^{\circ}\right)$ position from the opposite surface, where the surface-opening vertical flaw is located, does not provide very good results. In this position the ultrasonic wave hits first the flaw tip. The tip of a natural flaw is tight and does not provide significant reflection surface. Instead, the flaw may diffract quite a big portion of the ultrasonic energy, reflecting back only a smaller amount of it. Larger tilt angles provide higher echo amplitudes, when the fracture surface and the opening corner become more "visible" for the ultrasonic beam [1]. This is due to the combination of the raising amplitude of the corner echo and the fracture surface reflection and scattering.

The different incident beams may cause a self-shadowing phenomenon, which may occur when a wave is incident onto a rough surface at a sufficiently tilted angle. Then part of the surface is not directly "illuminated" by the incoming wave, but it is shaded by other parts of the surface. Self-shadowing spoils the phase coherence of the adjacent surface scattered waves and, thus, the amplitude of the overall scattered field may be diminished [13]. Occurrence of self-shadowing depends on the surface profile and is therefore difficult to take into account precisely.

In some cases a small flaw may produce higher response than a similarly tilted large flaw [2]. As a consequence, it is possible that when the flaw grows, repeated inspections would show decreasing signal amplitude. The degree of misorientation affects the strength of the diffracted ultrasonic signal from the flaw tip [18]. Figure 12 shows results obtained with three different $0^{\circ}$ probes (different wave modes and frequencies). All the graphs show that by changing the angle of the incident of ultrasonic wave, the obtained amplitude height may be remarkably changed.

\subsubsection{Effect of Oxide Film on Detection and Sizing}

During in-service inspection the tested flaw may be filled with air, water and/or have an oxide layer on its fracture 
Fig. 12 Pulse-echo response of a mechanical fatigue crack tip for (a) $5 \mathrm{MHz}$ longitudinal waves, (b) horizontally polarized $2.25 \mathrm{MHz}$ transverse waves and (c) vertically polarized $2.25 \mathrm{MHz}$ transverse waves. Solid lines show results of theoretical modelling and crosses are measured data [18]
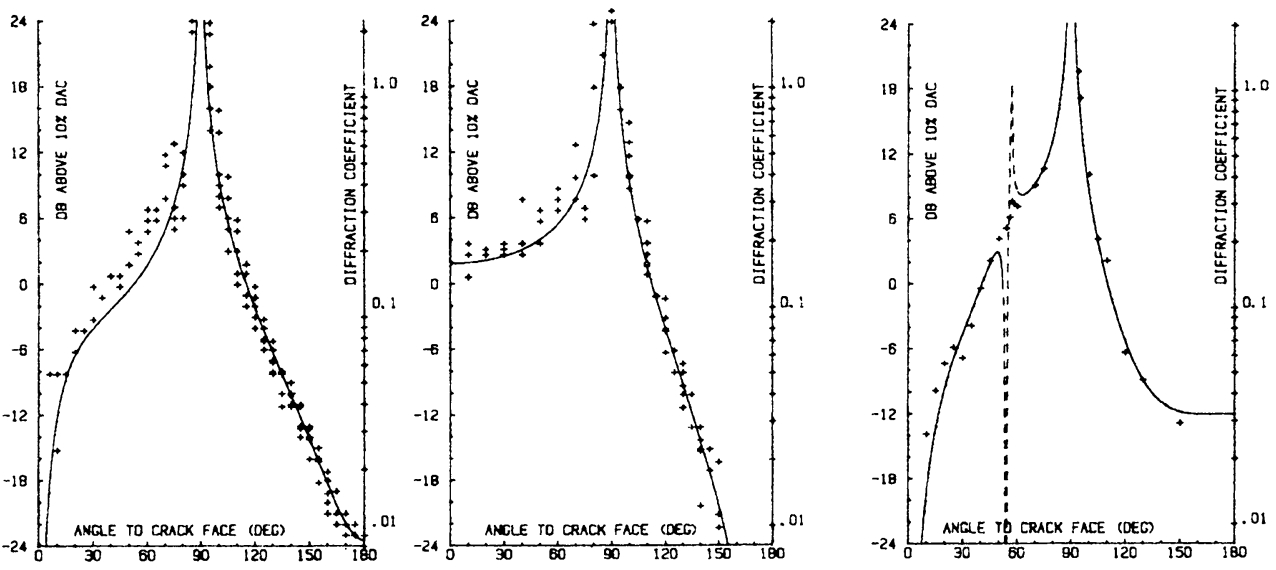

a)

b)

c) surfaces entailing different acoustic impedances and, hence, affecting the reflection and transmission of ultrasound. According to Crutzen et al. [4], the presence of corrosion products in the flaw enhances its transparency during ultrasonic inspection decreasing detectability. Filling the crack with oxide or water results in higher sensitivity to amplitude drop, when the crack is closed as with an air-filled crack [2]. That is, the transmission of ultrasound through the crack occurs earlier if the crack is filled with oxide or water. The sensitivity to tightness is ranked to be highest with water-filled cracks, second highest with oxide-filled cracks, while metallic, air-filled cracks are the least sensitive. The theoretical calculations (pulse-echo, $45^{\circ}, 4 \mathrm{MHz}$ ) of Temple [17] show similarly that a narrow (opening $2 \mu \mathrm{m}$ ) water-filled flaw gives $9.6 \mathrm{~dB}$ lower signal than air-filled one. Similar drop is obtained with a $4 \mu \mathrm{m}$ wide flaw in $2 \mathrm{MHz}$ inspection.

Some authors report opposite results from their studies. For example, Iida et al. [11] reported that if an oxide layer is present on the fracture surfaces, the changes in the ultrasonic echo heights, when closing the crack, are remarkably reduced. This was explained as a cause of air present in the crack hindering changes of the reflection coefficient of the sound pressure. Iida et al. [11] made a conclusion from their results that, even if compressive stresses are present in a component during the shutdown of a plant, the oxide films present on the fracture surfaces do not reduce the detectability of cracks.

During flaw sizing the oxide film grown on the fracture surfaces affects the sizing capability. This is due to the oxide layer on the fracture surfaces holding the metallic surfaces separate. The difference in the impedances of the metal and the oxide affects the obtained ultrasonic amplitude from the oxide filled flaws, as shown, e.g., by Iida et al. [11].

\subsubsection{Summary of Crack Characteristics Essential for UT}

In summary, the characteristics relevant for crack representativeness for UT techniques in general are:
1. location and orientation of the crack,

2. size of the crack,

3. opening of the crack through the whole path and at crack tip,

4. fracture surface roughness,

5. filling of the crack with some substance (e.g., water or oxide).

\section{Measurement Methods for Crack Characteristics}

A consistent set of measurement methods is needed to facilitate meaningful comparison of crack characteristics between service-induced cracks and various artificial cracks. While the importance and effect of several crack characteristics on NDE has been studied in the open literature (see paragraph 3 ), in most of these studies the parameters were not directly measured on actual service induced cracks. The first comprehensive framework for measuring crack characteristics for NDE was published by Wåle et al. [22, 23]. He measured comprehensive set of available images from serviceinduced cracks and, to facilitate this work, defined a set of measurement methods to be used. To date, this remains the most comprehensive data published on the crack characteristics of service-induced cracks. It must be noted, that the raw material, from which this information was extracted, was often of bad quality. The images were from failure analysis reports and taken to clarify reasons for failure - not to describe cracking for in-service inspection. Furthermore, all the measurements were done manually from paper images. Consequently, the measurements were rather laborious and partly inaccurate. This limited the possible measurement methods as well as the number of measured cracks.

In 2007, Trueflaw developed a more advanced set of measurement methods to overcome some of the limitations in the original Wåle methods resulting from the limitations mentioned above. This development was done in connection to a national development project where (among other 
Fig. 13 Crack opening at surface data from service induced cracks. Bars show measured range and line shows median value. Values from [22, 23]

Fig. 14 Crack opening at midway data from service induced cracks. Bars show measured range and line shows median value. Values from Wåle $[22,23]$

Fig. 15 Crack opening close to crack tip data from service induced cracks. Bars show measured range and line shows median value. Values from Wåle $[22,23]$

Fig. 16 Crack branching data from service induced cracks. Bars show measured range and line shows median value. Values from Wåle [22, 23]
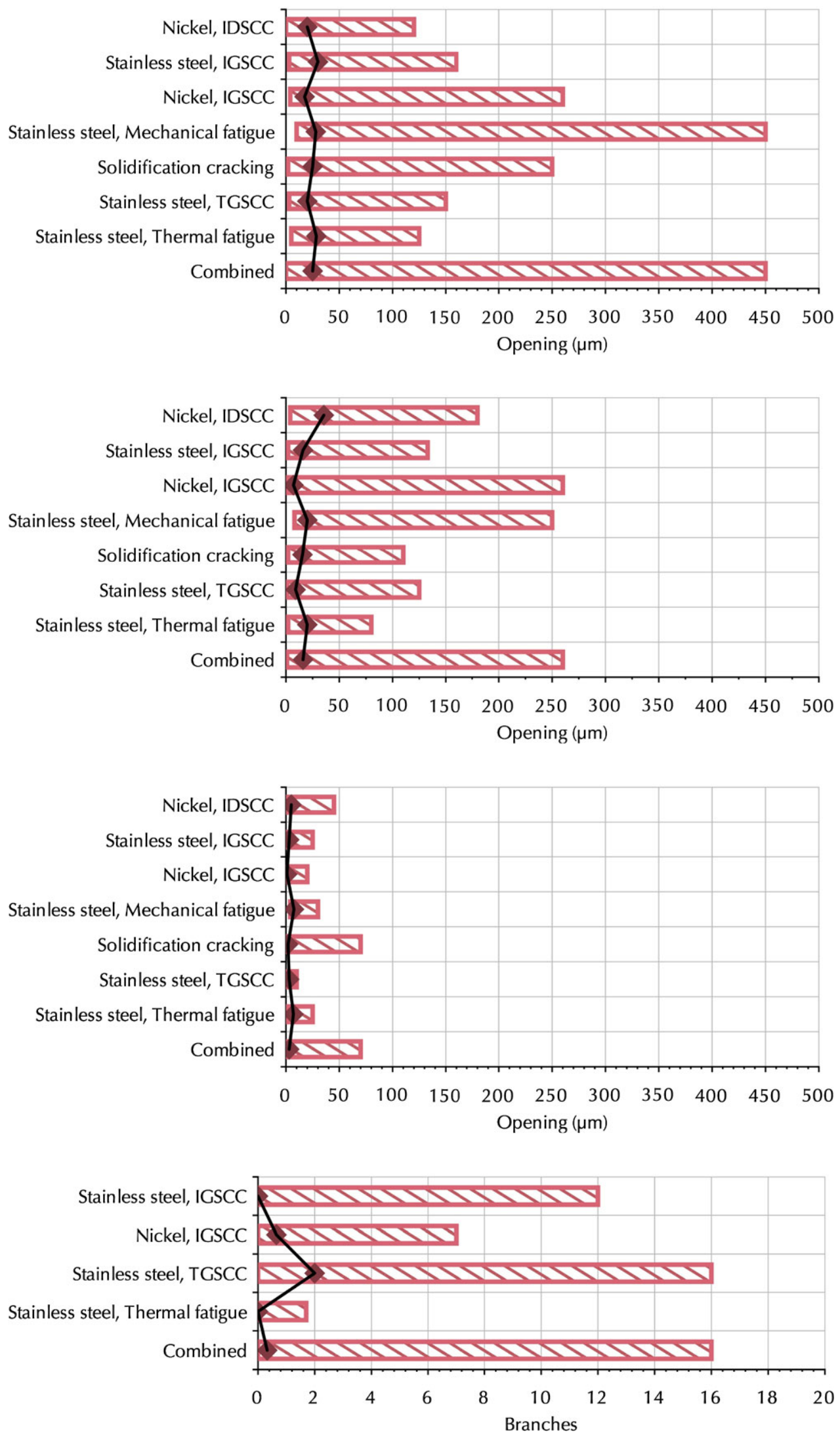
Fig. 17 Crack correlation length data from service induced cracks. Bars show measured range and line shows median value. Values from Wåle [22, 23]

Fig. 18 Crack intersections data from service induced cracks. Bars show measured range and line shows median value. Values from Wåle [22, 23]

Fig. 19 Crack surface roughness data from service-induced cracks. Bars show measured range and line shows median value. Values from Wåle [22, 23]

Fig. 20 Crack turns/mm data from service-induced cracks. Bars show measured range and line shows median value. Values from Wåle [22, 23]
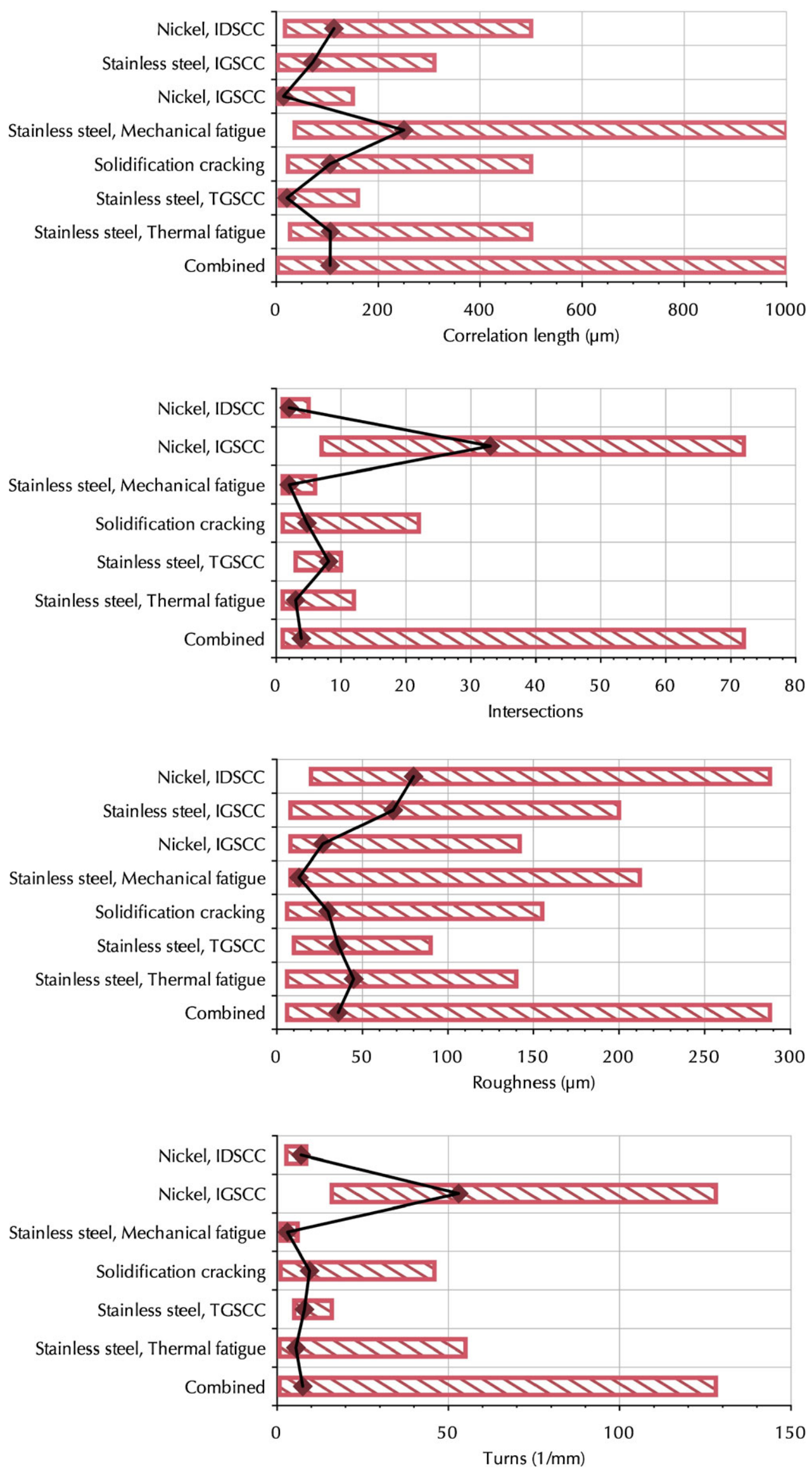
aims) a wide set of different artificially produced cracks were characterized in an effort to provide dataset on artificial crack characteristics comparable to the Wåle data [14]. Care was taken to retain comparability to the Wåle measurements in all possible measurements. Simultaneously, the measurement methods were developed to be more suited on automatic measurements with much improved repeatability and accuracy than what could have been reached manually. This measurement methodology is described in more detail in the Appendix.

This methodology (where applicable) is currently used by Trueflaw to document all manufactured cracks and it is presented here as a proposal for unified set of measurement methods for crack characteristics. It should be noted, that not all the measured characteristics presented may not be equally significant for NDE; some were included in the original Wåle reports for other purposes (e.g. leak-rate estimation) and they are included for completeness.

\section{Available Data on Service-Induced Cracks}

To this date, the most comprehensive published set of quantitative data on crack characteristics of service-induced cracks remains the work by Wåle [22, 23]. The data is revisualized here for easy reference in Figs. 13-20.

\section{Process for Confirming Artificial Flaw Representativeness}

A procedure for confirming and demonstrating representativeness of selected artificial flaws is outlined the following:

\section{Determine essential parameters.}

Consult paragraph 3 and inspection vendors to determine set of essential crack characteristics for the current inspection method. If uncertainty exists on whether a certain parameter is essential, it should be included to be conservative.

2. Check relevant data from service-induced cracks.

Assign target values and/or range for essential parameters based on available data from service-induced cracks. Data from paragraph 5 can be used.

3. Confirm that measured data from artificial cracks conforms to assigned criteria.

Crack characteristics for Trueflaw cracks can be obtained from manufacturing documentation. If the essential characteristics from used artificial cracks match the criteria, the representativeness is confirmed. Otherwise, the discrepancy must be justified by, e.g. technical justification.

Open Access This article is distributed under the terms of the Creative Commons Attribution Noncommercial License which permits any noncommercial use, distribution, and reproduction in any medium, provided the original author(s) and source are credited.

\section{Appendix: Measurement Methods for Crack Characteristics}

This annex describes the methods used by Trueflaw to measure various crack characteristics from digital images. These methods were developed to be comparable with the methods used by Wåle [23] but to overcome some of the limitations in these methods.

\section{A.1. Crack Detection, Centerline and Opening Measurements}

Measurements are done on digital image. The image plane is selected so, that the long side of the image corresponds to $0^{\circ}$ orientation of the crack. Measurement is done for each vertical image line. The line is smoothed, and background subtracted. The lowest brightness point is considered to be the crack. If this lowest point (signal) exceeds the mean deviation from background (noise) by a certain limit, it is considered a valid crack. The $\mathrm{S} / \mathrm{N}$ limit is adjustable and reported for each measurement individually. The crack edges are then measured from the unsmoothed brightness curve as width of the half-maximum. The crack centerline is the arithmetic mean between the crack edges. The image resolution (number of measurement lines per millimeter) is reported for each measurement image.

\section{A.2. Crack Roughness, Intersections and Correlation Length}

For these measurements, the crack length is divided to sections with a typical length of $1.5 \mathrm{~mm}$ (Wåle [23] used 1$2 \mathrm{~mm}$ ). For each section, a line is fitted to the crack centerline data obtained. Fitting is done by the least squares method. For each line, the roughness values $R_{a}$ and $R_{z}$ as well as number of intersections, and simplified correlation length are calculated. The $R_{a}$ is calculated according to following formula:

$R_{a}=\frac{\sum|e|}{l}$,

where $e$ is the difference between the fitted line and crack centerline and $l$ is the measurement length.

The $R_{z}$ is calculated according to following formula:

$R_{z}=\frac{\sum_{i=1}^{5} p_{i}-\sum_{j=1}^{5} v_{j}}{5}$,

where

$p_{i}$ is the $i$ th highest peak on the measurement length and $v_{j}$ is the $j$ th lowest valley on the measurement length. 
The number of intersections is defined as a point, where two preceding lines are below the fitted line and two following lines above it or vice versa. The correlation length is defined as the length of the measurement range divided by twice the number of intersections.

\section{A.3. Crack Turns}

To calculate the number of macroscopic turns in the crack, the crack centerline curve is divided to sections being typically $0.5 \mathrm{~mm}$. For each section, a line is fitted with the least square method. Whenever the angle between successive lines differs by more than $30^{\circ}$, a turn in the crack is identified.

\section{A.4. Orientation}

To calculate the crack orientation, a line is fitted to the crack centerline curve with the least squares method. The reported orientation is the angle of this line against the picture orientation.

\section{A.5. Macroscopic Shape, Branching, Microstructure, Discontinuities}

Macroscopic shape, branching, microstructure and discontinuities are manually calculated from the image with the same terms and methods as used by Wåle [23]. The shape is determined visually by one of the words: straight, winding, bend, bilinear or branched. The reported value for branching is the number of branches (greater than five grain diameters) per mm crack length. Microstructure is described with, e.g., one of the words: equi-axed grains, column formed grains (weld metal), cold worked, cast microstructure. The value reported for discontinuities is the number of discontinuities manually counted for the image.

\section{References}

1. Ahmed, S.R., Saka, M.: A sensitive ultrasonic approach to NDE of tightly closed small cracks. J. Press. Vessel Technol. 120, 384-392 (1998)

2. Becker, F.L., Doctor, S.R., Heasler, P.G., Morris, C.J., Pitman, S.G., Selby, G.P., Simonen, F.A.: Integration of NDE reliability and fracture mechanics-Phase I report. NUREG/CR-1696 PNL3469 , vol. 1,170 p. (1981)

3. Crutzen, S.J., Jehenson, P., Nichols, R.W., McDonald, N.: The major results of the PISC II RRT. Nucl. Eng. Des. 115, 7-21 (1989)

4. Crutzen, S., Lemaitre, P., Iacono, I.: Realistic defects suitable for ISI capability evaluation and qualification. In: Proceedings of the 14th International Conference on NDE in the Nuclear and Pressure Vessel Industries, 24-26 September 1996, Stockholm, Sweden, pp. 153-163 (1996)

5. Denby, D., Duncumb, A.C.: The effects of stress on the ultrasonic detectability of defects. In: Proceedings of the Conference of Nondestructive Testing in the Fitness-for-Purpose Assessment of Welded Constructions, pp. 73-81. The Weldiung Institute, Cambridge (1984)
6. Edwards, R., Gruber, G., Watson, P.: Fabrication of performance demonstration initiative specimens with controlled flaws. In: Proceedings on 13th International Conference on NDE in the Nuclear and Pressure Vessel Industries, 22-25 May 1995, Kyoto, Japan, pp. 167-176 (1995)

7. Edwards, R.L., Watson, P.D., Gruber, G.J.: Fabrication of specimens with controlled flaws for procedure development and personnel training and qualification. In: Proceedings on 12th International Conference on NDE in the Nuclear and Pressure Vessel Industries, 11-13 October 1993, Philadelphia, Pennsylvania, USA (1993), pp. 93-100

8. Gauthier, V.: Thermal fatigue cracking of safety injection system pipes non destructive testing inspections feedback. In: Proceedings of NEA/CSNI Specialists' Meeting on: Experiences with Thermal Fatigue in LWR Piping Caused by Mixing and Stratification, 8-10 June, Paris, France (1998), pp. 436-453

9. Green, E.R.: Worst-case defects affecting ultrasonic inspection reliability. Mater. Eval. 47, 1401-1407 (1989)

10. Ibrahim, S.I., Whittaker, V.N.: The influence of crack topography and compressive stresses on the ultrasonic detection of fatigue cracks in submerged arc welds. Br. J. NDT, September, pp. 233240 (1981)

11. Iida, K., Takumi, K., Naruse, A.: Influence of stress condition on flaw detectability and sizing accuracy by ultrasonic inspection. In: The Ninth International Conference on Nondestructive Evaluation in the Nuclear Industry, 25-28 April, Tokyo, Japan, pp. 563-567 (1988)

12. Kemppainen, M., Virkkunen, I., Packalén, T., Sillanpää, J., Paussu, R.: Importance of crack opening in UT inspection qualification. In: Proceedings of the 6th International Conference on NDE in Relation to Structural Integrity for Nuclear and Pressurised Components, 8-19 October, Budapest, Hungary, p. 93105 (2007)

13. Ogilvy, J.A.: Model for the ultrasonic inspection of rough defects. Ultrasonics 27, 69-79 (1989)

14. Packalén, T., Sillanpää, J., Kemppainen, M., Virkkunen, I., Paussu, R.: The influence of the crack opening in the UT inspection qualification. In: Proceedings of the 6th International Conference on NDE in Relation to Structural Integrity for Nuclear and Pressurised Components, 8-10 October, Budapest, Hungary, pp. 463-470 (2007)

15. Pirson, J., Roussel, G.: Emergency core cooling system pipe crack incident at the Tihange unit 1 plant. In: Proceedings of NEA/CSNI Specialists' Meeting on: Experiences with Thermal Fatigue in LWR Piping Caused by Mixing and Stratification, 8-10 June, Paris, France, pp. 103-114 (1998)

16. Saka, M., Fukuda, Y.: NDT of closed cracks by ultrasonic propagation along the crack surface. NDT E Int. 24(4), 191-194 (1991)

17. Temple, J.A.G.: The effects of stress and crack morphology on time-of-flight diffraction signals. Int. J. Press. Vessels Piping 19, 185-211 (1985)

18. Toft, M.W.: Experimental studies of ultrasonic reflection from various types of misoriented defect. In: Proceedings of 21st Annual British Conference on Non-Destructive Testing - NDT, vol. 86, 193-206 (1986)

19. Waites, C., Whittle, J.: The status of performance demonstration and evaluation developments. Insight 40(12), 810-813 (1998)

20. Wirdelius, H., Osterberg, E.: Study of defect characteristics essential for NDT testing methods ET, UT and RT. SKI Project Number 98267, SKI Report 00:42, October 2000, Sweden, 50 p. (2000)

21. Wirdelius, H.: Probe model implementation in the null field approach to crack scattering. J. Nondestruct. Eval. 11(1), 29-39 (1992) 
22. Wåle, J., Ekström, P.: Crack Characterisation for In-service Inspection Planning, SKI Projekt 14.4-940389, 94164 SAQ/FoURapport 95/70, SAQ Kontroll AB, Stockholm, Sweden, 84 p. (1995)

23. Wåle, J.: Crack characterization for in-service inspection planning - an update. SKI reference 14.43-200543105, ISRN SKI-R-06/24SE, SKI, Stockholm, Sweden (2006)
24. Yoneyama, H., Senoo, M., Miharada, H., Uesugi, N.: Comparison of echo heights between fatigue crack and EDM notch. In: Proceedings of 2nd International Conference on NDE in Relation to Structural Integrity for Nuclear and Pressurized Components, 2426 May, 2000, New Orleans, Louisiana, USA, 8 p. (2000) 